MAN/HEP/2009/32

arXiv:0909.1749

September 2009

\title{
Strangephilic Higgs Bosons in the MSSM
}

\author{
J. S. Lee ${ }^{a}$, Y. Peters ${ }^{b}$, A. Pilaftsis ${ }^{b}$ and C. Schwanenberger ${ }^{b}$ \\ ${ }^{a}$ Physics Division, National Center for Theoretical Sciences, Hsinchu, Taiwan \\ ${ }^{b}$ School of Physics and Astronomy, University of Manchester, \\ Manchester M13 9PL, United Kingdom
}

\begin{abstract}
We suggest a new CPX-derived scenario for the search of strangephilic MSSM Higgs bosons at the Tevatron and the LHC, in which all neutral and charged Higgs bosons decay predominantly into pairs of strange quarks and into a strange and a charm quark, respectively. The proposed scenario is realized within a particular region of the MSSM parameter space and requires large values of $\tan \beta$, where threshold radiative corrections are significant to render the effective strange-quark Yukawa coupling dominant. Experimental searches for neutral Higgs bosons based on the identification of $b$-quark jets or $\tau$ leptons may miss a strangephilic Higgs boson and its existence could be inferred indirectly by searching for hadronically decaying charged Higgs bosons. Potential strategies and experimental challenges to search for strangephilic Higgs bosons at the Tevatron and the LHC are discussed.
\end{abstract}




\section{Introduction}

The search strategy for Higgs bosons at high-energy colliders, such as LEP, the Tevatron, and the LHC, depends crucially on their decay properties, and especially on the strength of their couplings to the kinematically allowed decaying particles [1 5]. In the Standard Model (SM), the Higgs boson Yukawa couplings to fermions are directly proportional to the fermion masses, i.e.

$$
h_{f}^{\mathrm{SM}}=\frac{\sqrt{2} m_{f}}{v}
$$

where $v \simeq 246 \mathrm{GeV}$ is the vacuum expectation value (VEV) of the SM Higgs doublet. Thus, the bottom-quark Yukawa coupling is suppressed, for example, by the factor $m_{b} / m_{t}$ compared to the top-quark one. However, in extensions of the SM with more than one Higgs doublet, the above relation (1) between the fermion mass and its Yukawa coupling gets modified. In the so-called minimal supersymmetric standard model (MSSM) which involves two Higgs doublets, one has at the tree-level

$$
h_{f=u}^{\mathrm{MSSM}}=\frac{\sqrt{2} m_{u}}{v \sin \beta}, \quad h_{f=d, l}^{\mathrm{MSSM}}=\frac{\sqrt{2} m_{d, l}}{v \cos \beta},
$$

where $\tan \beta=\sin \beta / \cos \beta$ denotes the ratio of the VEVs of the two Higgs doublets and $u, d, l$ stand for the up- and down-type quarks and charged leptons, respectively. In this case, the bottom-quark Yukawa coupling $h_{b}$ can be as large as the top-quark one $h_{t}$ for large values of $\tan \beta \sim m_{t} / m_{b}$. In contrast, the ratios of the Yukawa couplings among the down-type quarks and charged leptons remain the same as in the SM:

$$
\frac{h_{d, s}^{\mathrm{MSSM}}}{h_{b}^{\mathrm{MSSM}}}=\frac{h_{d, s}^{\mathrm{SM}}}{h_{b}^{\mathrm{SM}}}=\frac{m_{d, s}}{m_{b}}, \quad \frac{h_{e, \mu}^{\mathrm{MSSM}}}{h_{\tau}^{\mathrm{MSSM}}}=\frac{h_{e, \mu}^{\mathrm{SM}}}{h_{\tau}^{\mathrm{SM}}}=\frac{m_{e, \mu}}{m_{\tau}} .
$$

As a consequence, the relative branching ratios of the neutral and charged Higgs bosons into light fermions do not alter, e.g. $B\left(H_{1,2,3} \rightarrow s \bar{s}\right) / B\left(H_{1,2,3} \rightarrow b \bar{b}\right) \ll 1$ and $B\left(H^{+} \rightarrow\right.$ $c \bar{s}) / B\left(H^{+} \rightarrow \tau^{+} \nu_{\tau}\right) \ll 1$ for large values of $\tan \beta$.

In this paper we propose a new benchmark scenario for the search of neutral and charged Higgs bosons, $H_{1,2,3}$ and $H^{ \pm}$, at the Tevatron and the LHC. In this scenario all the neutral and charged Higgs bosons decay predominantly into strange quarks and into a strange and a charm quarks, respectively. As we will show in Section 2, this scenario occurs within a restricted area of the MSSM parameter space characterized by large values of $\tan \beta$, where threshold radiative corrections are significant to give rise to a sizeable effective strange-quark Yukawa coupling $h_{s}$ of order 1*. Because of their strong affinity to strange quarks, we call these Higgs scalars strangephilic. The particular strangephilic

*We note that our scenario differs from that in [6] where some of the Higgs bosons may favorably decay into light quarks. In our case, instead, all charged and neutral Higgs bosons decay predominantly to strange quarks thanks to a large effective $h_{s}$. 
Higgs scenario, which we study in detail in Section 3, is characterized by the fact that $B\left(H_{1,2,3} \rightarrow s \bar{s}\right) / B\left(H_{1,2,3} \rightarrow b \bar{b}\right) \gtrsim 1$ and $B\left(H^{+} \rightarrow c \bar{s}\right) / B\left(H^{+} \rightarrow \tau^{+} \nu_{\tau}\right) \gtrsim 1$ and by large decay widths of order $10 \mathrm{GeV}$ for the Higgs bosons $H_{1,2,3}$ and $H^{ \pm}$. In Section 4 , we discuss potential strategies and the associated experimental challenges to look for strangephilic neutral and charged Higgs bosons at the Tevatron and the LHC. Finally, the main results of our analysis are summarized in Section 5 .

\section{Strangephilic Higgs Bosons}

In the MSSM, the tree-level relations between the masses and couplings given in (2) get significantly modified by finite loop-induced threshold corrections mediated by the exchange of gluinos and charginos [7]. For the down-type quark Yukawa couplings, such a modification becomes important for large values of $\tan \beta$. More explicitly, at large $\tan \beta$ the down-type quark Yukawa couplings take on the form

$$
h_{q}=\frac{\sqrt{2} m_{q}}{v \cos \beta} \frac{1}{1+\Delta_{q} \tan \beta}
$$

where $q=d, s, b$ and $\Theta$

$$
\begin{aligned}
\Delta_{d} & =\frac{2 \alpha_{s}}{3 \pi} \mu^{*} M_{3}^{*} I\left(M_{\tilde{Q}_{1}}^{2}, M_{\tilde{D}_{1}}^{2},\left|M_{3}\right|^{2}\right), \\
\Delta_{s} & =\frac{2 \alpha_{s}}{3 \pi} \mu^{*} M_{3}^{*} I\left(M_{\tilde{Q}_{2}}^{2}, M_{\tilde{D}_{2}}^{2},\left|M_{3}\right|^{2}\right), \\
\Delta_{b} & =\frac{2 \alpha_{s}}{3 \pi} \mu^{*} M_{3}^{*} I\left(M_{\tilde{Q}_{3}}^{2}, M_{\tilde{D}_{3}}^{2},\left|M_{3}\right|^{2}\right)+\frac{\left|h_{t}\right|^{2}}{16 \pi^{2}} \mu^{*} A_{t}^{*} I\left(M_{\tilde{Q}_{3}}^{2}, M_{\tilde{U}_{3}}^{2},|\mu|^{2}\right),
\end{aligned}
$$

with the loop function $I(a, b, c)$ defined as

$$
I(a, b, c)=\frac{a b \ln (a / b)+b c \ln (b / c)+a c \ln (c / a)}{(a-b)(b-c)(a-c)} .
$$

Considering $\alpha_{s} \sim 0.1$ and $\left|h_{t}\right| \sim 1$, the gluino-loop contribution to $\Delta_{b}$ is estimated to be larger than the chargino-loop one by the factor $\sim \pi|\mu|^{2} /\left|A_{t} M_{3}\right|$ when $|\mu|^{2},\left|M_{3}\right|^{2} \gg$ $M_{\tilde{Q}_{3}, \tilde{U}_{3}, \tilde{D}_{3}}^{2}$ [9]. In this case, assuming the universal sfermion masses or $M_{\tilde{Q}_{3}}=M_{\tilde{Q}_{2}}, M_{\tilde{U}_{3}}=$ $M_{\tilde{U}_{2}}$, and $M_{\tilde{D}_{3}}=M_{\tilde{D}_{2}}$, one has $\Delta_{s} \simeq \Delta_{b}$ leading to the same relation (3) as in the case without the inclusion of threshold corrections, i.e. $B\left(H_{1,2,3} \rightarrow s \bar{s}\right) / B\left(H_{1,2,3} \rightarrow b \bar{b}\right) \ll 1$ and $B\left(H^{+} \rightarrow c \bar{s}\right) / B\left(H^{+} \rightarrow \tau^{+} \nu_{\tau}\right) \ll 1$. However, for particular choices of the theoretical parameters, $\Delta_{s}$ can differ dramatically from $\Delta_{b}$. In particular, if $\left|1+\Delta_{s} \tan \beta\right| \rightarrow 0$ and $\left|1+\Delta_{b} \tan \beta\right| \sim \mathcal{O}(1)$ [10], this would result in a scenario with $\left|h_{s}\right| \gg\left|h_{b}\right|$, where all neutral Higgs bosons $H_{1,2,3}$ will decay predominantly into strange quarks and the charged Higgs scalars $H^{ \pm}$into strange and charm quarks.

\footnotetext{
†Throughout this work, we follow the conventions and notations of CPsuperH $[8]$.
} 
In our phenomenological analysis, we will utilize the effective Lagrangian approach to describe the interaction of the neutral and charged Higgs bosons to quarks in the presence of $\tan \beta$-enhanced threshold corrections. The effective Lagrangian is given by

$$
\mathcal{L}_{H q q^{\prime}}=-\sum_{i} g_{q} H_{i} \bar{q}\left(g_{H_{i} \bar{q} q}^{S}+i g_{H_{i} \bar{q} q}^{P} \gamma_{5}\right) q-\left[g_{q^{\prime} q} H^{+} \bar{q}^{\prime}\left(g_{H^{+} \bar{q}^{\prime} q}^{S}+i g_{H^{+} \bar{q}^{\prime} q}^{P} \gamma_{5}\right) q \quad+\text { h.c. }\right],
$$

where $q=d, s, b$ and $q^{\prime}=u, c, t$. At the tree level,

$$
g_{q}=\frac{g m_{q}}{2 M_{W}}, \quad g_{H_{i} \bar{q} q}^{S}=O_{\phi_{1} i} / \cos \beta, \quad g_{H_{i} \bar{q} q}^{P}=-\tan \beta O_{a i}
$$

for the neutral Higgs boson $H_{1,2,3}$ with the $3 \times 3$ mixing matrix $O$ such that $\left(\phi_{1}, \phi_{2}, a\right)^{T}=$ $O_{\alpha i}\left(H_{1}, H_{2}, H_{3}\right)^{T}$ with $M_{H_{1}} \leq M_{H_{2}} \leq M_{H_{3}}$. For the charged Higgs boson,

$g_{q^{\prime} q}=-\frac{g m_{q^{\prime}}}{\sqrt{2} M_{W}}, \quad g_{H^{+\bar{q}^{\prime} q}}^{S}=\frac{1}{2}\left[\frac{1}{\tan \beta}+\frac{m_{q}}{m_{q^{\prime}}} \tan \beta\right], \quad g_{H^{+\bar{q}^{\prime} q}}^{P}=\frac{i}{2}\left[\frac{1}{\tan \beta}-\frac{m_{q}}{m_{q^{\prime}}} \tan \beta\right]$.

In the presence of threshold corrections, the couplings $g_{H_{i} \bar{q} q}^{S}, g_{H_{i} \bar{q} q}^{P}, g_{H^{+} \bar{q}^{\prime} q}^{S}$ and $g_{H^{+} \bar{q}^{\prime} q}^{P}$ read [1]]:

$$
\begin{aligned}
g_{H_{i} \bar{q} q}^{S}= & \operatorname{Re}\left(\frac{1}{1+\Delta_{q} \tan \beta}\right) \frac{O_{\phi_{1} i}}{\cos \beta}+\operatorname{Re}\left(\frac{\Delta_{q}}{1+\Delta_{q} \tan \beta}\right) \frac{O_{\phi_{2} i}}{\cos \beta} \\
& +\operatorname{Im}\left[\frac{\Delta_{q}\left(\tan ^{2} \beta+1\right)}{1+\Delta_{q} \tan \beta}\right] O_{a i}, \\
g_{H_{i} \bar{q} q}^{P}= & -\operatorname{Re}\left(\frac{\tan \beta-\Delta_{q}}{1+\Delta_{q} \tan \beta}\right) O_{a i}+\operatorname{Im}\left(\frac{\Delta_{q} \tan \beta}{1+\Delta_{q} \tan \beta}\right) \frac{O_{\phi_{1} i}}{\cos \beta} \\
& -\operatorname{Im}\left(\frac{\Delta_{q}}{1+\Delta_{q} \tan \beta}\right) \frac{O_{\phi_{2} i}}{\cos \beta}, \\
g_{H^{+} \bar{q}^{\prime} q}^{S}= & \frac{1}{2}\left[\frac{1}{\tan \beta}+\frac{m_{q}}{m_{q^{\prime}}} \frac{\tan \beta-\Delta_{q}^{*}}{1+\Delta_{q}^{*} \tan \beta}\right] \\
g_{H^{+} \bar{q}^{\prime} q}^{P}= & \frac{i}{2}\left[\frac{1}{\tan \beta}-\frac{m_{q}}{m_{q^{\prime}}} \frac{\tan \beta-\Delta_{q}^{*}}{1+\Delta_{q}^{*} \tan \beta}\right] .
\end{aligned}
$$

For completeness, the charged Higgs boson couplings to leptons are given by

$$
g_{\nu_{l} l}=-\frac{g m_{l}}{\sqrt{2} M_{W}}, \quad g_{H^{+} \bar{\nu}_{l} l}^{S}=\tan \beta / 2, \quad g_{H^{+} \bar{\nu}_{l} l}^{P}=-i \tan \beta / 2 .
$$

In the limit of $\left|1+\Delta_{s}^{*} \tan \beta\right| \rightarrow 0$, it is not difficult to show that $\left|g_{H_{1,2,3} \bar{s} s}^{S, P}\right| \gg\left|g_{H_{1,2,3} \bar{b} b}^{S, P}\right|$ and $\left|g_{H^{+} \bar{c} s}^{S, P}\right| \gg\left|g_{H^{+} \bar{\nu}_{\tau} \tau}^{S, P}\right|$. For Higgs bosons lighter than the top quark, this possibility leads to a strangephilic scenario where the neutral and charged Higgs bosons will decay predominantly into $s \bar{s}$ and $c \bar{s}$, respectively, instead of $b \bar{b}$ and $\tau^{+} \nu_{\tau}$. In our numerical analysis, we have included the effects of the mass splitting in third generation squarks and all other threshold corrections that are not enhanced by $\tan \beta[8]$. 
Finally, it is important to remark that the proposed strangephilic scenario will generically induce large flavour-changing-neutral-current (FCNC) effects mediated by Higgs bosons at large $\tan \beta$, because of the inherent hierarchy between the first two and third generations [10]. One possible way to avoid these large FCNC effects would be to go beyond the framework of minimal Supergravity (mSUGRA) and allow for sizeable flavour-mixing effects in the squark sector, such that a kind of cancellation mechanism becomes operative [10]. Evidently, FCNC effects strongly depend on the strength of the off-diagonal effective Yukawa couplings and the flavour structure of the model in general. We will not address this highly model-dependent issue in the present work. Our interest here is to analyze the implications of a large diagonal strange-quark effective Yukawa coupling, independently of the off-diagonal ones, for Higgs-boson searches at the Tevatron and the LHC.

\section{Strangephilic Higgs Benchmark Scenarios}

As strangephilic viable models, we consider the following two benchmark scenarios derived from CPX [12]:

$$
\begin{array}{ll}
\mathbf{L}: & M_{\tilde{Q}_{3}}=M_{\tilde{U}_{3}}=M_{\tilde{D}_{3}}=0.5 \mathrm{TeV} ; M_{\tilde{L}_{3}}=M_{\tilde{E}_{3}}=0.7 \mathrm{TeV}, \\
& M_{H^{ \pm}}=130 \mathrm{GeV}, \quad|\mu|=2 \mathrm{TeV}, \quad\left|A_{t, b, \tau}\right|=1 \mathrm{TeV}, \quad\left|M_{3}\right|=1 \mathrm{TeV}, \\
& \Phi_{A_{t}}=\Phi_{A_{b}}=\Phi_{A_{\tau}}=90^{\circ}, \quad \Phi_{3}=180^{\circ}, \\
& \rho_{\tilde{Q}}=\rho_{\tilde{U}}=\rho_{\tilde{L}}=\rho_{\tilde{E}}=1, \\
& 1 \leq \tan \beta \leq 120, \quad 2 \leq \rho_{\tilde{D}} \leq 6, \\
\mathbf{S}: \quad & M_{\tilde{Q}_{3}}=M_{\tilde{D}_{3}}=2 \mathrm{TeV} ; M_{\tilde{U}_{3}}=M_{\tilde{L}_{3}}=M_{\tilde{E}_{3}}=1 \mathrm{TeV}, \\
& M_{H^{ \pm}}=120 \mathrm{GeV}, \quad|\mu|=2 \mathrm{TeV},\left|A_{t, b, \tau}\right|=1 \mathrm{TeV}, \quad\left|M_{3}\right|=1 \mathrm{TeV}, \\
& \Phi_{A_{t}}=\Phi_{A_{b}}=\Phi_{A_{\tau}}=90^{\circ}, \quad \Phi_{3}=180^{\circ}, \\
& \rho_{\tilde{U}}=\rho_{\tilde{L}}=\rho_{\tilde{E}}=1, \\
& 1 \leq \tan \beta \leq 60, \quad 0.1 \leq \rho_{\tilde{Q}, \tilde{D}} \leq 0.6 .
\end{array}
$$

In the above, we have introduced the $\rho_{\tilde{X}}$ parameters to account for a mass hierarchy between the first two and third generations:

$$
M_{\tilde{X}_{1,2}}=\rho_{\tilde{X}} M_{\tilde{X}_{3}}
$$

with $\tilde{X}=\tilde{Q}, \tilde{U}, \tilde{D}, \tilde{L}, \tilde{E}$. In the $\mathbf{L}$ scenario, the Higgs bosons exhibit a strangephilic behaviour for large values of $\rho_{\tilde{D}}$ greater than 1 , whereas in the $\mathbf{S}$ scenario the strangephilic nature of the Higgs bosons is realized for small values of $\rho_{\tilde{Q}, \tilde{D}}$ less than 1 . Finally, the remaining parameters are fixed as

$$
\begin{aligned}
& \left|M_{1}\right|=250 \mathrm{GeV}, \quad \Phi_{1}=0^{\circ} ; \quad\left|M_{2}\right|=500 \mathrm{GeV}, \quad \Phi_{2}=0^{\circ} ; \\
& \left|A_{e, \mu, u, c, d, s}\right|=\left|A_{t, b, \tau}\right|, \quad\left|\Phi_{A_{e, \mu, u, c, d, s}}\right|=0^{\circ}, \quad \Phi_{\mu}=0 .
\end{aligned}
$$


In the upper-left frame of Fig. 1, the blue and red regions show where the strangequark Yukawa coupling is larger than the bottom-quark Yukawa one, which is obtained by simultaneously varying the hierarchy factor $\rho_{\tilde{D}}=M_{\tilde{D}_{1,2}} / M_{\tilde{D}_{3}}$ and $\tan \beta$. The unshaded region is not theoretically allowed, as it leads to a non-perturbative value for the strangequark Yukawa coupling where $\left|h_{s}\right|>2$. The larger $\rho_{\tilde{D}}$ is, the larger value for $\tan \beta$ is needed to satisfy the limit $\left|1+\Delta_{s}^{*} \tan \beta\right| \rightarrow 0$ and so realize the strangephilic condition: $\left|h_{s}\right|>\left|h_{b}\right|$. In the upper-right frame, we show the absolute values of the bottom- and strange-quark and the tau-lepton Yukawa couplings as functions of $\tan \beta$, for $\rho_{\tilde{D}}=4.6$. In the low $\tan \beta$ region we always have $\left|h_{s}\right| \ll\left|h_{b}\right|$. As $\tan \beta$ increases, the bottomquark Yukawa coupling grows rapidly and, in the region between $\tan \beta=12$ and 41, results in tachyonic bottom squarks or in a Higgs-boson mass matrix with a complex or negative eigenvalue. For larger values of $\tan \beta$, in the region $80 \lesssim \tan \beta \lesssim 100$, one can have $\left|h_{s}\right|>\left|h_{b}\right|$, where $\left|1+\Delta_{s}^{*} \tan \beta\right| \simeq 0$. The unshaded region in the upper-left frame corresponds to the interval $88 \lesssim \tan \beta \lesssim 92$, within which $\left|h_{s}\right|>2$ lies beyond the realm of validity of perturbation theory. We note that in the high $\tan \beta$ region, we can always have $\left|h_{s}\right|>\left|h_{b}\right|$ for any value of $\tan \beta$ by appropriately tuning the free parameter $\rho_{\tilde{D}}$ as shown in the upper-left frame.

In the lower-left frame of Fig. 1, we show the three neutral Higgs boson masses as functions of $\tan \beta$ together with the $110.6 \mathrm{GeV}$ (95\% C.L.) experimental bound on the flavor-independent hadronically decaying SM Higgs boson derived from LEP [13] 3 . For large values of $\tan \beta$, the lightest Higgs boson lies below the experimental bound but escapes the LEP searches due to its small couping $g_{H_{1} V V}^{2} \lesssim 0.02$, if $\tan \beta \gtrsim 65$, as shown in the lower-right frame. In this kinematic region, the coupling of $H_{2}$ to the gauge bosons is large, $g_{H_{2} V V}^{2} \gtrsim 0.97$. However, for $80 \lesssim \tan \beta \lesssim 100$, the $H_{2}$ boson is predominantly strangephilic with a mass $M_{H_{2}} \gtrsim 110 \mathrm{GeV}$, so it remains undetected thanks to the flavourindependent LEP limit mentioned above. For $\tan \beta \gtrsim 100$, the $H_{2}$ boson is heavier than $\sim 114 \mathrm{GeV}$, thereby satisfying the absolute LEP bound on the SM Higgs boson. Thus, for $\tan \beta \gtrsim 80$ and $\rho_{\tilde{D}}>4$, any strangephilic region is compatible with the LEP limits in the $\mathbf{L}$ benchmark scenario (13). In this respect, we should comment that the existence of uncertainties due to possible variations of the top-quark mass, the higher-order quantum corrections and the known field-theoretic differences [15] between the Feynmandiagrammatic and RG-improved approaches may result in significant uncertainties in the predictions for the MSSM Higgs-boson mass spectrum, which are typically bigger than $\sim 3 \mathrm{GeV}$. Therefore, given all these different sources of uncertainties, the constraints derived here on the Higgs-boson masses and their couplings to the $\mathrm{Z}$ boson using the public

$\ddagger$ This constraint is close to the limit for which the Yukawa coupling becomes non-perturbative at the renormalization-group scale of $1-2 \mathrm{TeV}$, i.e. $\left|h_{s}\right|^{2} / 4 \pi>1$.

$\S$ Conservatively, we require that the mass of a strangephilic Higgs boson be larger than $110.6 \mathrm{GeV}$, when its coupling to the $Z$ boson is larger than about 0.1 of the SM $H Z Z$-coupling. A more precise treatment of the LEP and TEVATRON limits might be obtained by using the public code HiggsBounds [14. 
code CPsuperH, which implements the RG-improved approach, should be regarded as fair and conservative.

One can obtain a similar LEP2-compatible strangephilic scenario for smaller values of $\tan \beta$ in the $\mathbf{S}$ benchmark scenario (14), for $\rho_{\tilde{Q}, \tilde{D}}<1$. Specifically, the upper-left frame of Fig. 2 shows that $\left|h_{s}\right|$ can be larger than $\left|h_{\tau}\right|$ for $30 \lesssim \tan \beta \lesssim 55$, when the free parameter $\rho_{\tilde{Q}, \tilde{D}}$ is tuned to a relative narrow region around a particular value between $\sim 0.3$ and $\sim 0.5$. For instance, in the upper-right frame of Fig. 2, we see that $\left|h_{s}\right|$ is dominant within the narrow region: $37 \lesssim \tan \beta \lesssim 40$, for the specific value of $\rho_{\tilde{Q}, \tilde{D}}=0.4$. We note that for $\tan \beta \gtrsim 30$ and $\rho_{\tilde{Q}, \tilde{D}} \gtrsim 0.3$, the two lighter Higgs bosons cannot be detected by LEP searches, because $g_{H_{1,2} V V}^{2} \lesssim 0.01$, as can be seen from the lower frames of Fig. 2, Moreover, the mass of the heaviest neutral Higgs state $H_{3}$ is always beyond the current LEP limit.

Even though the proposed benchmark scenarios are not generic within the MSSM, the degree of parameter tuning required for realizing strangephilic Higgs bosons is not excessive. As we will see in the next section, there is a significant range of $\tan \beta$ values, for which strangephilic Higgs bosons can occur within the $\mathbf{L}$ and $\mathbf{S}$ benchmark scenarios.

We conclude this section by noticing that both the $\mathbf{L}$ and $\mathbf{S}$ scenarios require large CP phases for the $A$ terms, i.e. $\Phi_{A_{t}}=\Phi_{A_{b}}=\Phi_{A_{\tau}}=90^{\circ}$, in order to satisfy the flavourindependent LEP bound [13]. These large CP phases together with the large $|\mu|=2\left|A_{t, b, \tau}\right|$ are the characteristic features of the CPX scenario [12]. There are severe constraints on the size of the CP-odd phases which arise from the non-observation of the Thallium, neutron, and Mercury electric dipole moments (EDMs). Specifically, we note that the enhanced strange-quark Yukawa coupling may induce large neutron EDM in the Parton Quark Model and large Higgs-mediated EDMs. Nevertheless, these constraints could, in principle, be evaded in CPX-like scenarios, by arranging for cancellations among the different one- and two-loop contributions to EDMs [11, 16].

\section{Potential Search Strategies and Experimental Challenges}

In this section, we analyze the generic phenomenological features of the strangephilic Higgs bosons in the $\mathbf{L}$ and $\mathbf{S}$ scenarios. We also discuss potential search strategies and experimental challenges to look for strangephilic neutral and charged Higgs scalars.

Let us first consider the neutral Higgs sector. Figure 3 shows our numerical results for the three neutral Higgs bosons in the $\mathbf{L}$ scenario. We observe that it is always possible to choose a LEP2-compatible $\rho_{\tilde{D}}$ to realize a strangephilic scenario for any large value of $\tan \beta>80$. Choosing $\rho_{\tilde{D}}=4.6$, we display in Fig. 3 the dependence of the branching ratios (left frames) and the total decay widths (right frames) on $\tan \beta$. We note the large total 
decay widths of the order of a few $\mathrm{GeV}$ or larger where $B\left(H_{1,2,3} \rightarrow s \bar{s}\right)>B\left(H_{1,2,3} \rightarrow b \bar{b}\right)$ and/or $B\left(H_{1,2,3} \rightarrow s \bar{s}\right)>B\left(H_{1,2,3} \rightarrow \tau^{+} \tau^{-}\right)$. The width of the $H_{2}$ boson is not as enhanced as those of $H_{1,3}$ and remains below $0.3 \mathrm{GeV}$, since the $H_{2}$ boson is most likely $\phi_{2}$, which does not couple to the down-type quarks and the charged leptons at the tree level.

Figure 4 shows our numerical results in the $\mathbf{S}$ benchmark scenario, for $\rho_{\tilde{Q}, \tilde{D}}=0.4$. In this case, one can get a strangephilic scenario around $\tan \beta \sim 38$; see the upper-right frame of Fig. 2. As before, we observe that the decay widths of the $H_{1,2}$ bosons are large $\mathcal{O}(10) \mathrm{GeV}$, whereas the $H_{3}$ width $\Gamma_{H_{3}}$ remains below $0.3 \mathrm{GeV}$, since the $H_{3}$ boson is most likely $\phi_{2}$ in this scenario.

The strangephilic region, $80 \lesssim \tan \beta \lesssim 100(35 \lesssim \tan \beta \lesssim 42)$ for the $\mathbf{L}(\mathbf{S})$ scenario with $\rho_{\tilde{D}}=4.6\left(\rho_{\tilde{Q}, \tilde{D}}=0.4\right)$, is determined by the condition that $B\left(H_{1,2,3} \rightarrow s \bar{s}\right)$ is bigger than $B\left(H_{1,2,3} \rightarrow b \bar{b}\right)$ or bigger than $B\left(H_{1,2,3} \rightarrow \tau^{+} \tau^{-}\right)$. At the Tevatron and the LHC, strangephilic neutral Higgs bosons can be substantially produced through the fusion of strange quarks: $s \bar{s} \rightarrow H_{1,2,3}$. The Higgs bosons produced in the $s$-channel would decay into $s$ quark and, without any efficient tagging technique for the identification of $s$-quark jets, the observation of strangephilic neutral Higgs bosons is experimentally challenging, if not impossible, unless the neutral Higgs bosons have sizeable branching ratios to photons. In particular, conventional searches at the Tevatron or the LHC that rely on tagging of $b$-quark jets or the identification of $\tau$ leptons will miss a strangephilic neutral Higgs boson.

Alternatively, one may consider searching for strangephilic neutral Higgs bosons in associate production channels, such as $g g \rightarrow t \bar{t} H_{1,2,3}, q q \rightarrow q q H_{1,2,3}$ and $q q^{(\prime)} \rightarrow V H_{1,2,3}$, with $V=Z, W^{ \pm}$. However, conventional searches at the Tevatron and the LHC for such processes assume that the neutral Higgs bosons decay predominantly into $b \bar{b}, \tau^{+} \tau^{-}$, or $W^{+} W^{-}$. Again, without an efficient $s$-quark jet tagging identification, the light-quark multijet background will render the sensitivity of the conventional search strategies to those channels rather problematic. One viable option might be to perform a flavour-independent analysis of the central exclusive diffractive Higgs production, $p p \rightarrow p+H_{1,2,3}+p$, which could help to determine the mass and the broad width of the strangephilic neutral Higgs bosons, provided $H_{1,2,3}$ are produced at sufficiently high rates.

Let us now turn our attention to the charged Higgs boson. Figures 5 and 6 show numerical results for the charged Higgs boson in the $\mathbf{L}$ and $\mathbf{S}$ scenarios, respectively. Like the neutral Higgs bosons, strangephilic charged Higgs bosons can be copiously produced through the fusion of strange and charm quarks, $c \bar{s} \rightarrow H^{+}$. But, their observation would be challenging, requiring the detection of $s$ and $c$ quarks. Unlike the neutral Higgs bosons, however, light strangephilic charged Higgs bosons can be searched for in the $t \bar{t}$ production channel at the Tevatron and the LHC, where the top quarks decay subsequently into $H^{ \pm}$ and $b$-quarks, provided $B\left(t \rightarrow H^{+} b\right)$ is substantial [17. Including threshold corrections, 
the partial decay width $t \rightarrow H^{+} b$ is given by

$$
\Gamma\left(t \rightarrow H^{+} b\right)=\frac{g_{t b}^{2} m_{t}}{16 \pi}\left(\left|g_{H^{+} \bar{t} b}^{S}\right|^{2}+\left|g_{H^{+} \bar{t} b}^{P}\right|^{2}\right)\left(1-\frac{M_{H^{ \pm}}^{2}}{m_{t}^{2}}\right)^{2} .
$$

As shown in Fig. 7, the branching ratio $B\left(t \rightarrow H^{+} b\right)$ is smaller than $10 \%$ in the $\mathbf{L}$ scenario, whilst it can be much larger in the $\mathbf{S}$ scenario depending on the actual value of $\tan \beta$.

We note that conventional searches for charged Higgs bosons that analyze only the tauonic decay channel $H^{+} \rightarrow \tau^{+} \nu$ loose their sensitivity in particular regions of $\tan \beta$. This happens, for example, if $\tan \beta$ is in the region between $\sim 80$ and $\sim 100$ in the $\mathbf{L}$ benchmark scenario (see Fig. 5, left panel) or between $\sim 35$ and $\sim 42$ in the $\mathbf{S}$ benchmark scenario (see Fig. 6, left panel). In both cases, the charged Higgs boson decays predominantly into $c$ and $s$ quarks. In order to cover the full range of $\tan \beta$ without missing the possible existence of a strangephilic charged Higgs boson, both decay channels, $H^{+} \rightarrow c \bar{s}$ and $H^{+} \rightarrow \tau^{+} \nu$, need to be investigated.

From an experimental point of view, searches for hadronically decaying charged Higgs bosons, such as strangephilic charged Higgs bosons, have two major advantages over the search for tauonically decaying charged Higgs bosons.

First, the detection is already possible through the ratios of the $t \bar{t}$ production cross sections in dileptonic, semi-leptonic and all-hadronic final states. Compared to the number of $t \bar{t}$ pairs in semi-leptonic (dileptonic) final states, a strangephilic charged Higgs boson would lead to a larger number of $t \bar{t}$ pairs in all-hadronic (semi-leptonic) final states than predicted by the SM. Measurements of such cross-section ratios will be available in a relative early stage of the LHC, since large systematic uncertainties cancel in the ratio. Recently, the D0 collaboration [17] was first to use cross-section ratios to obtain information on charged Higgs bosons. In contrast, tauonic charged Higgs decays lead to a disappearance in all those channels. Therefore, they will be harder to detect them without a reliable understanding of $\tau$-lepton identification which is experimentally challenging.

Second, since a strangephilic charged Higgs boson decays into two jets, its full invariant mass can be reconstructed with a much better resolution than in tauonic decays of the charged Higgs boson, where only the missing transverse momentum of the $\tau$-neutrino can be used for the mass reconstruction. This will lead to a larger sensitivity in the search for strangephilic charged Higgs bosons. A first search for charged Higgs bosons decaying into charm and strange quarks with full reconstruction of the charged Higgs mass was carried out recently by the CDF collaboration [19]. This recent CDF analysis can exclude only a very narrow region of the $\mathbf{S}$ scenario around $\tan \beta \simeq 38$, provided the mass of the charged Higgs boson is small enough.

IThe calculation of the top-quark branching ratios has been implemented in the most recent version of CPsuperH2. 0 by including the $\mathcal{O}\left(\alpha_{s}\right)$ QCD corrections [18], as well as the threshold corrections. 


\section{Conclusions}

We have analyzed a new benchmark scenario that can be realized within the MSSM, for which the strength of the $s$-quark Yukawa coupling relative to the $b$-quark one can be dramatically enhanced through gluino-mediated one-loop corrections. In particular, for large values of $\tan \beta$ and for a certain choice of the soft SUSY-breaking mass parameters, one can obtain a strangephilic scenario in which the neutral and charged Higgs bosons have dominant or substantial decay modes into a pair of strange quarks and into a charm and a strange quark, respectively. Even though not fully included in the present analysis, we note that as well as third generation quarks and squarks, strange squarks may also contribute significantly to the Higgs-boson mass spectrum through a large effective strange-quark Yukawa coupling at the loop level.

At the Tevatron collider and the LHC, strangephilic neutral and charged Higgs bosons can be substantially produced through $s$-channel fusion processes initiated by strange and charm quarks. In general, their experimental observation would be challenging without any efficient tagging technique for the identification of $s$-quark jets. As was discussed in Section 4, for the neutral Higgs bosons, alternative production channels may be considered, such as exclusive diffractive Higgs production, which could be helpful in experimental searches. On the other hand, it proves easier to detect strangephilic charged Higgs bosons $H^{ \pm}$, if produced at sufficiently high rate, e.g. in top-quark decays. In particular, investigating both the decay channels $H^{+} \rightarrow \tau^{+} \nu$ and $H^{+} \rightarrow c \bar{s}$ proves an important search

strategy for detecting a strangephilic charged Higgs boson [20. A positive signal in these channels would point towards an entire strangephilic Higgs sector, which could be realized even within a constrained two-Higgs doublet model, such as the MSSM.

In our on-going quest of the thus-far elusive Higgs boson, we strongly encourage the experimental Collaborations at the Tevatron and the LHC to perform a detailed analysis and systematic search for strangephilic Higgs bosons.

\section{Acknowledgements}

We thank Un-ki Yang for a discussion concerning future experimental prospects for $s$-quark jet identification. The work of A.P. was supported in part by the STFC research grant: PP/D000157/1. The work of C.S. and Y.P. was supported by the Royal Society. 


\section{References}

[1] R. Barate et al. [The LEP Working Group for Higgs boson searches, ALEPH, DELPHI, L3, and OPAL Collaborations], Phys. Lett. B 565 (2003) 61 arXiv:hep-ex/0306033.

[2] S. Schael et al. [The LEP Working Group for Higgs Boson Searches, ALEPH, DELPHI, L3, and OPAL Collaborations], Eur. Phys. J. C 47 (2006) 547 arXiv:hep-ex/0602042.

[3] M. S. Carena et al. [Higgs Working Group Collaboration], arXiv:hep-ph/0010338.

[4] G. Aad et al. [The ATLAS Collaboration], arXiv:0901.0512 [Unknown].

[5] G. L. Bayatian et al. [CMS Collaboration], J. Phys. G 34 (2007) 995.

[6] M. S. Carena, S. Mrenna and C. E. M. Wagner, Phys. Rev. D 62 (2000) 055008 arXiv:hep-ph/9907422;

M. S. Carena, S. Heinemeyer, C. E. M. Wagner and G. Weiglein, Eur. Phys. J. C 26 (2003) 601 arXiv:hep-ph/0202167.

[7] R. Hempfling, Phys. Rev. D 49 (1994) 6168;

L. J. Hall, R. Rattazzi and U. Sarid, Phys. Rev. D 50 (1994) 7048 arXiv:hep-ph/9306309;

M. S. Carena, M. Olechowski, S. Pokorski and C. E. M. Wagner, Nucl. Phys. B 426 (1994) 269 arXiv:hep-ph/9402253;

D. M. Pierce, J. A. Bagger, K. T. Matchev and R. j. Zhang, Nucl. Phys. B 491 (1997) 3 arXiv:hep-ph/9606211];

F. Borzumati, G. R. Farrar, N. Polonsky and S. D. Thomas, Nucl. Phys. B 555 (1999) 53 arXiv:hep-ph/9902443;

K. S. Babu and C. F. Kolda, Phys. Lett. B 451 (1999) 77 arXiv:hep-ph/9811308.

[8] J. S. Lee, A. Pilaftsis, M. Carena, S. Y. Choi, M. Drees, J. R. Ellis and C. E. M. Wagner, Comput. Phys. Commun. 156 (2004) 283 arXiv:hep-ph/0307377];

J. S. Lee, M. Carena, J. Ellis, A. Pilaftsis and C. E. M. Wagner, Comput. Phys. Commun. 180 (2009) 312 [arXiv:0712.2360 [hep-ph]].

[9] F. Borzumati, J. S. Lee and W. Y. Song, Phys. Lett. B 595 (2004) 347 arXiv:hep-ph/0401024.

[10] A. Dedes and A. Pilaftsis, Phys. Rev. D 67 (2003) 015012 arXiv:hep-ph/0209306.

[11] A. Pilaftsis, Nucl. Phys. B 644 (2002) 263 arXiv:hep-ph/0207277.

[12] M. Carena, J. R. Ellis, A. Pilaftsis and C. E. M. Wagner, Phys. Lett. B 495 (2000) 155 arXiv:hep-ph/0009212. 
[13] A. Heister et al. [ALEPH Collaboration], Phys. Lett. B 544 (2002) 25 arXiv:hep-ex/0205055];

G. Abbiendi et al. [OPAL Collaboration], Phys. Lett. B 597 (2004) 11 arXiv:hep-ex/0312042].

[14] P. Bechtle, O. Brein, S. Heinemeyer, G. Weiglein and K. E. Williams, arXiv:0811.4169 [hep-ph].

[15] M.S. Carena, H.E. Haber, S. Heinemeyer, W. Hollik, C.E.M. Wagner and G. Weiglein, Nucl. Phys. B 580 (2000) 29 arXiv:hep-ph/0001002].

[16] For a recent detailed analysis, see, J. R. Ellis, J. S. Lee and A. Pilaftsis, JHEP 0810 (2008) 049 [arXiv:0808.1819 [hep-ph]].

[17] V. M. Abazov et al. [D0 Collaboration], arXiv:0903.5525 [hep-ex]; D0 Collaboration, D0 Note 5715-CONF (2008).

[18] See, for example, K. G. Chetyrkin, R. Harlander, T. Seidensticker and M. Steinhauser, arXiv:hep-ph/9910339.

[19] CDF Collaboration, arXiv:0907.1269 [hep-ex].

[20] For a first experimental search for strangephilic charged Higgs bosons at Tevatron, see V. M. Abazov et al. [D0 Collaboration], arXiv:0908.1811 [hep-ex]. 

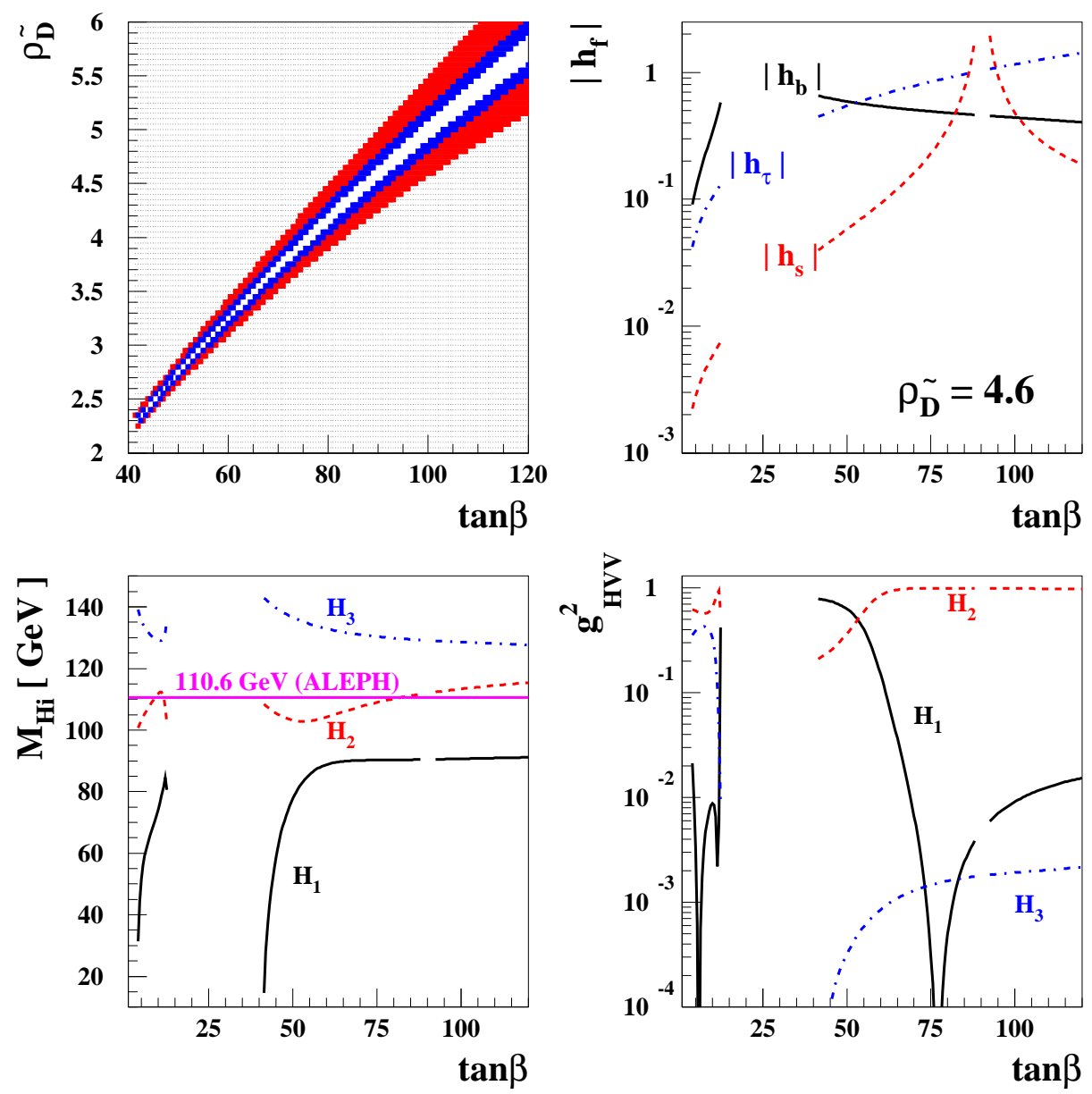

Figure 1: Numerical estimates in the $\mathbf{L}$ scenario defined in (13). The upper-left frame shows the region (blue and red), in which $\left|h_{s}\right| \geq\left|h_{b}\right|$ in the $\rho_{\tilde{D}}$-tan $\beta$ plane. The narrow unshaded region sandwiched between the shaded regions is not allowed theoretically resulting in a nonperturbative value $\left|h_{s}\right|>2$, while the red-and blue-shaded regions correspond to $\left|h_{s}\right| \leq 1$ and $1 \leq\left|h_{s}\right| \leq 2$, respectively. In the upper-right frame, the absolute values of the bottomand strange-quark and the tau-lepton Yukawa couplings are shown as functions of $\tan \beta$ in the solid, dashed and dash-dotted lines, respectively, assuming $\rho_{\tilde{D}}=4.6$. The region between $\tan \beta=12$ and 41 is not allowed theoretically giving rise to tachyonic bottom squarks or to a Higgs boson mass matrix with a complex or negative eigenvalue. The lower frames display the masses and their couplings to the $W$ or $Z$ bosons $(V=W, Z)$ as functions of $\tan \beta$. 

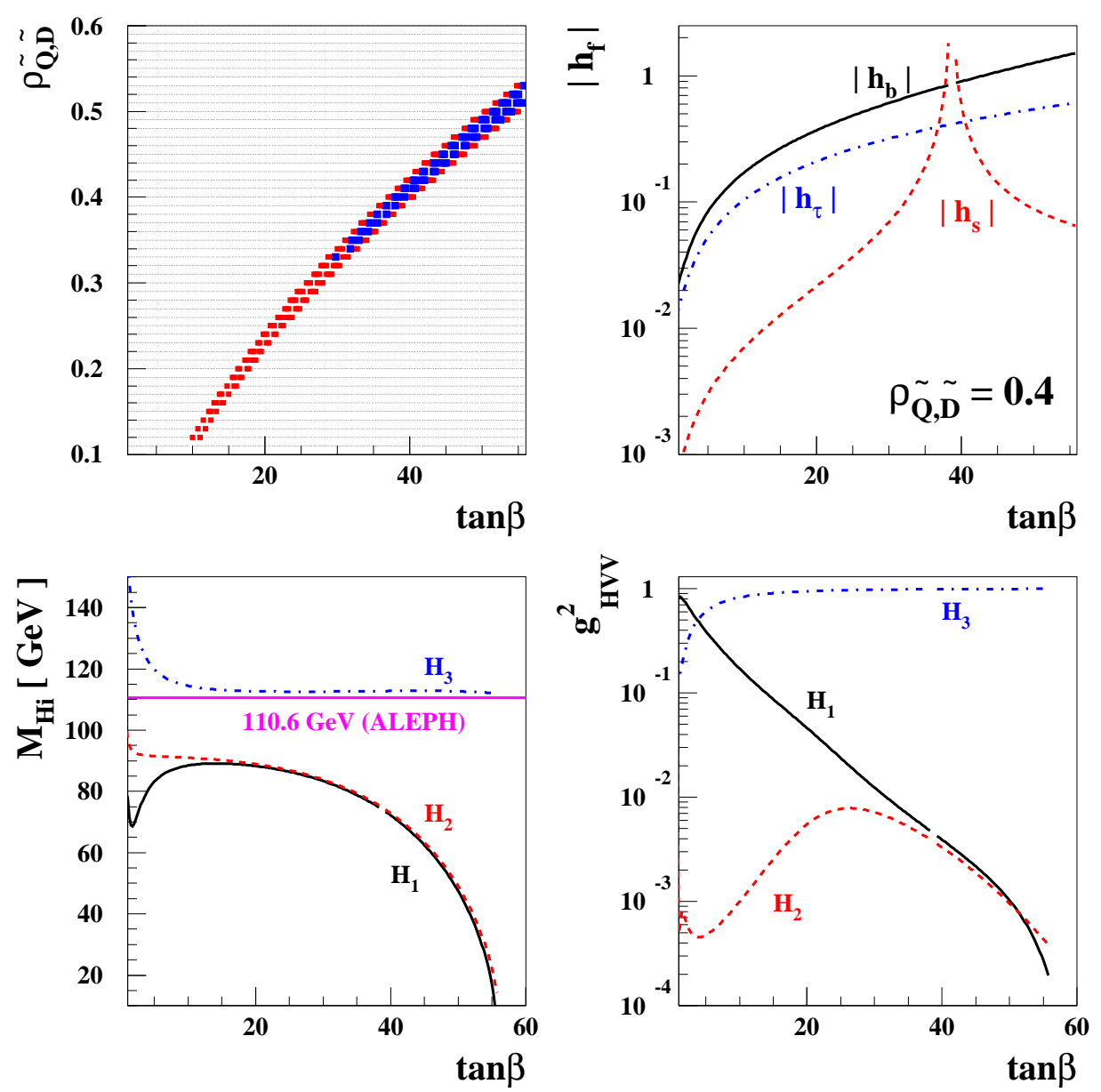

Figure 2: The same as in Fig. 团, but for the $\mathbf{S}$ scenario defined in (14). The upper left frame shows the region, for which $\left|h_{s}\right| \geq\left|h_{\tau}\right|$. The upper-right frame displays the dependence of the absolute values of the Yukawa couplings $\left|h_{s, b, \tau}\right|$ on $\tan \beta$, for $\rho_{\tilde{Q}, \tilde{D}}=0.4$. 

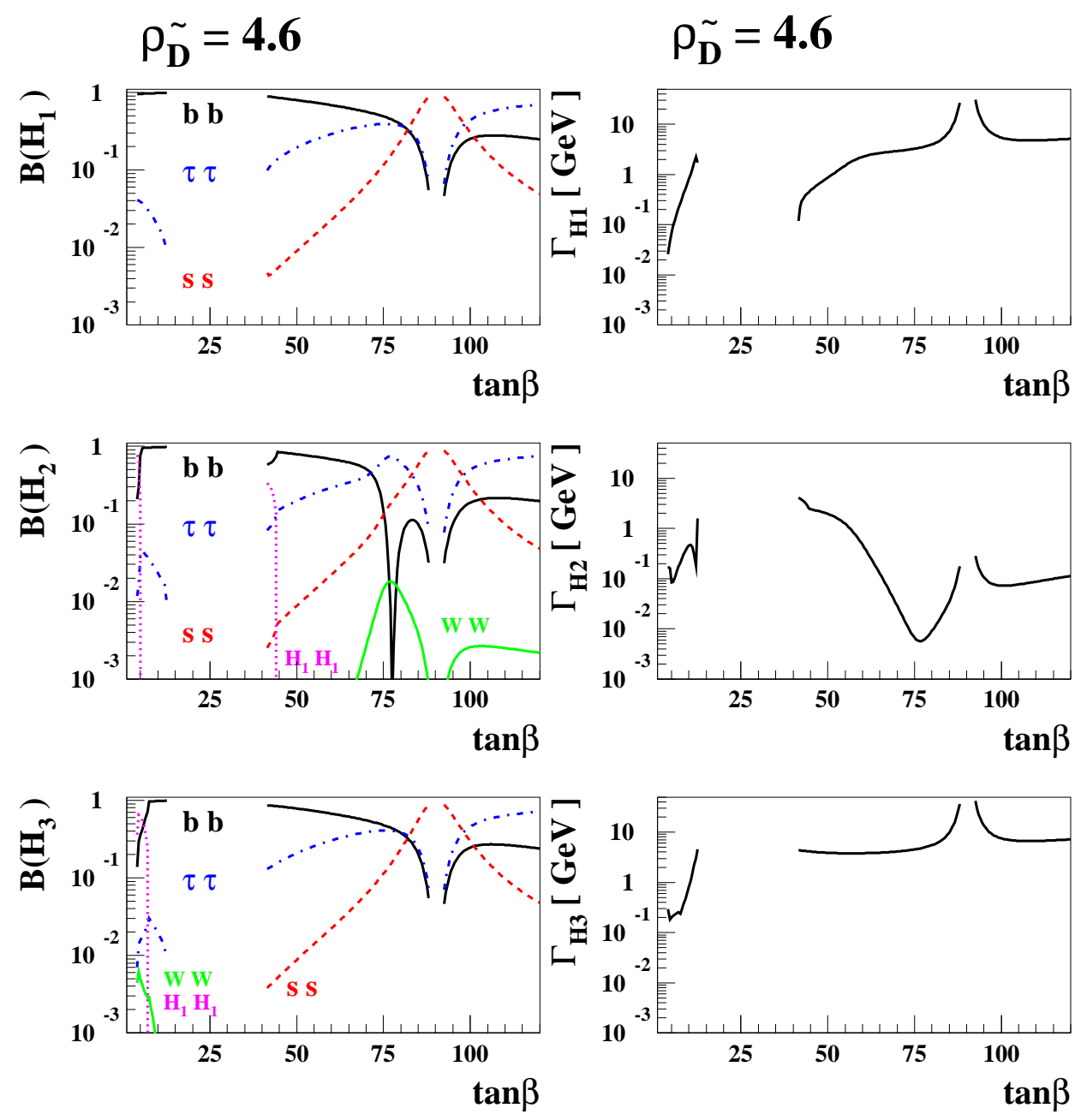

Figure 3: Numerical estimates of branching ratios (right) and total decay widths (left) of the three neutral Higgs bosons $H_{1,2,3}$ as functions of $\tan \beta$, for $\rho_{\tilde{D}}=4.6$, in the $\mathbf{L}$ scenario given in (13). 

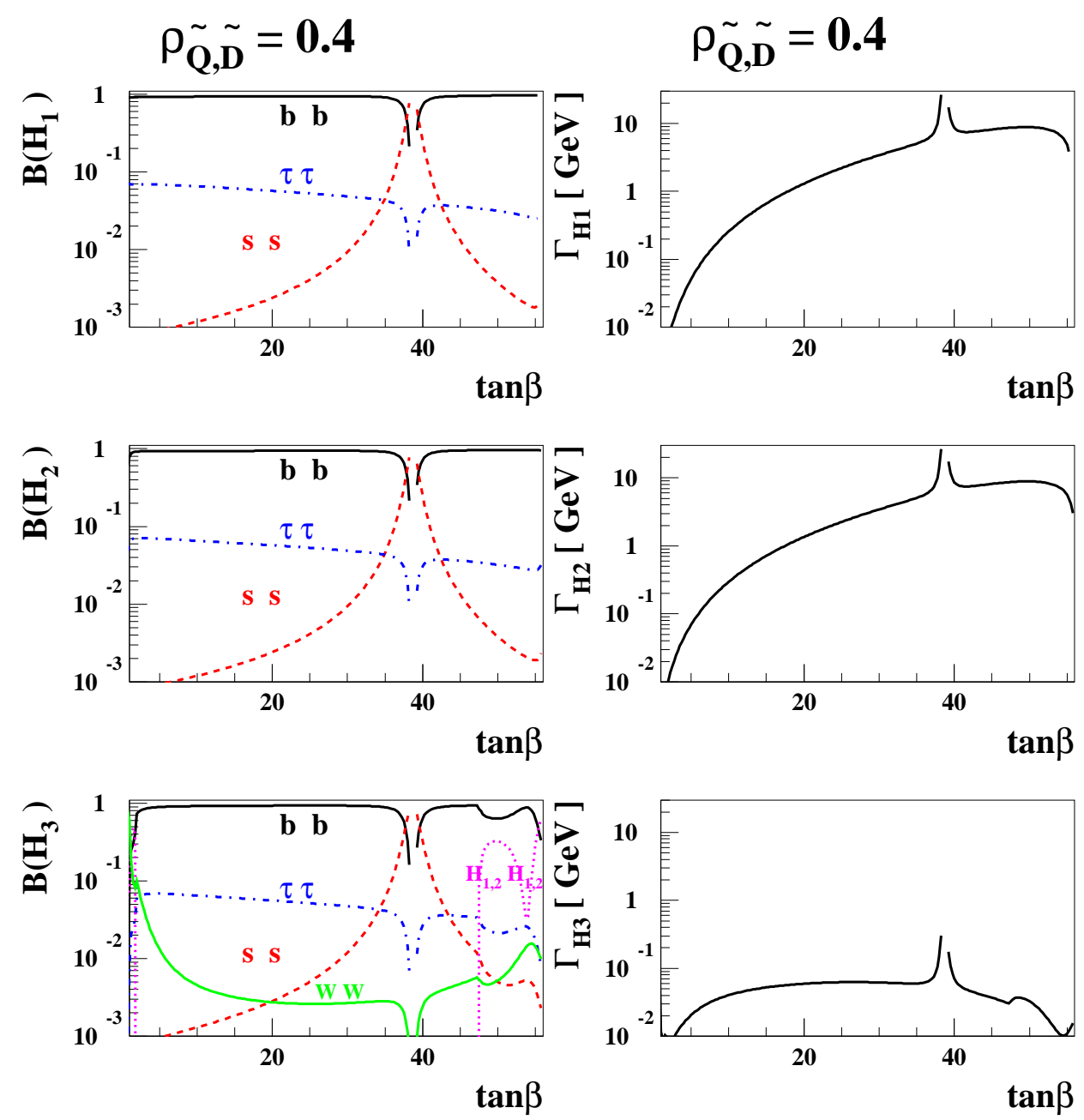

Figure 4: Numerical estimates of branching ratios (right) and total decay widths (left) of the three neutral Higgs bosons $H_{1,2,3}$ as functions of $\tan \beta$, for $\rho_{\tilde{Q}, \tilde{D}}=0.4$, in the $\mathbf{S}$ scenario given in (14). 

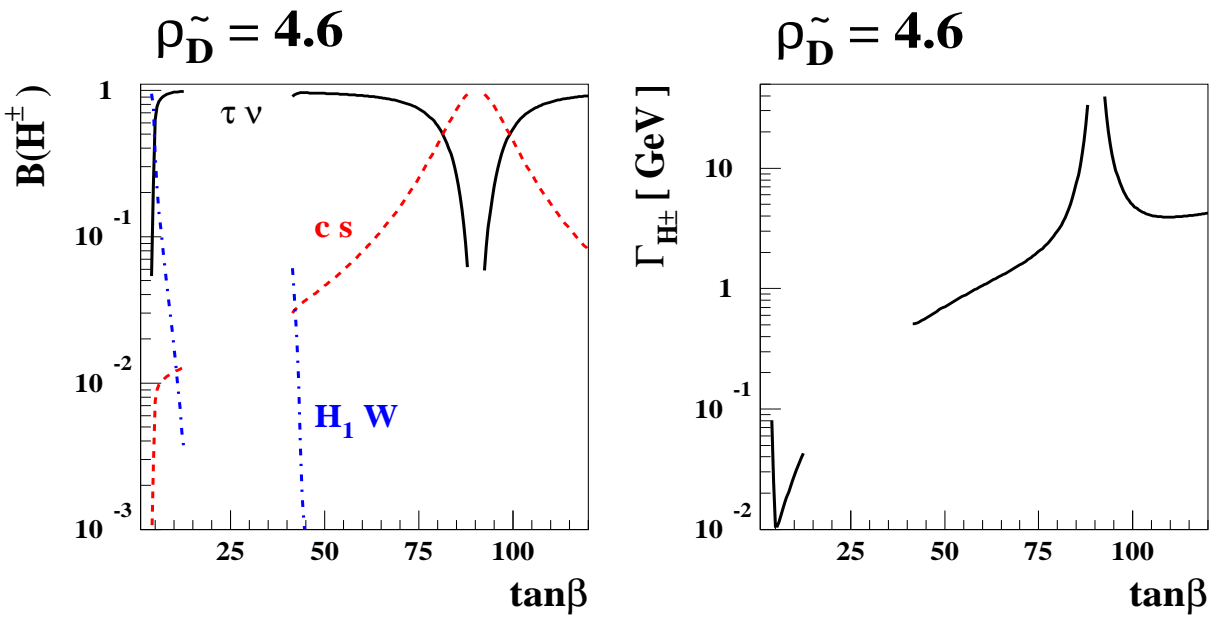

Figure 5: The same as in Fig. 3 but for the charged Higgs boson.
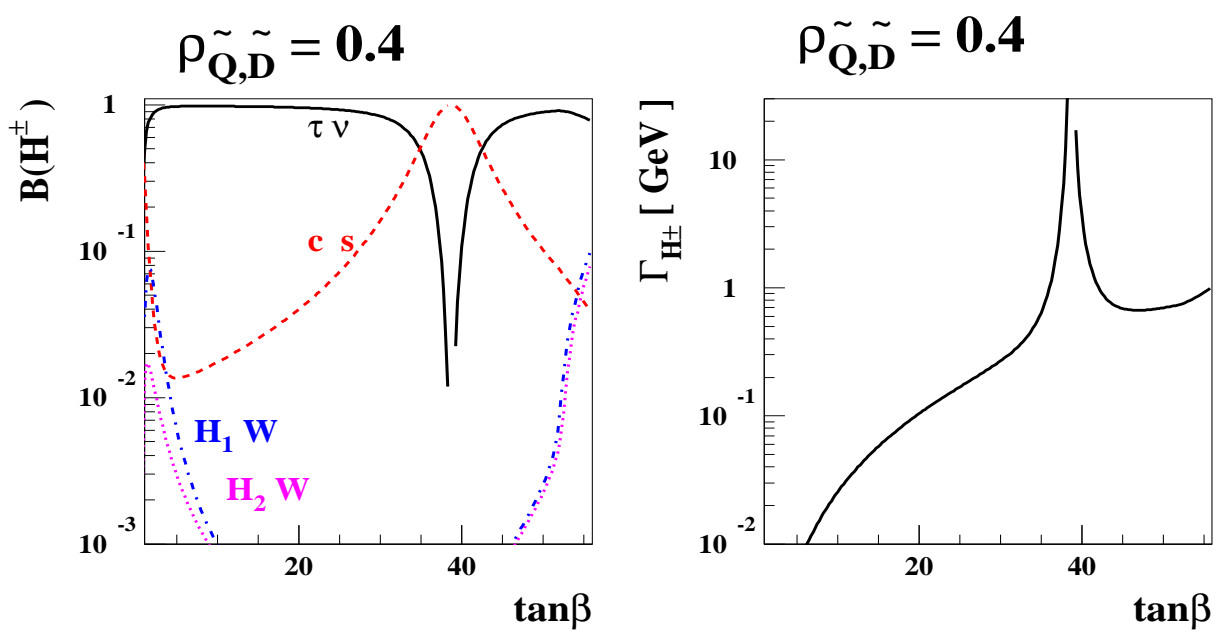

Figure 6: The same as in Fig. 4 but for the charged Higgs boson. 

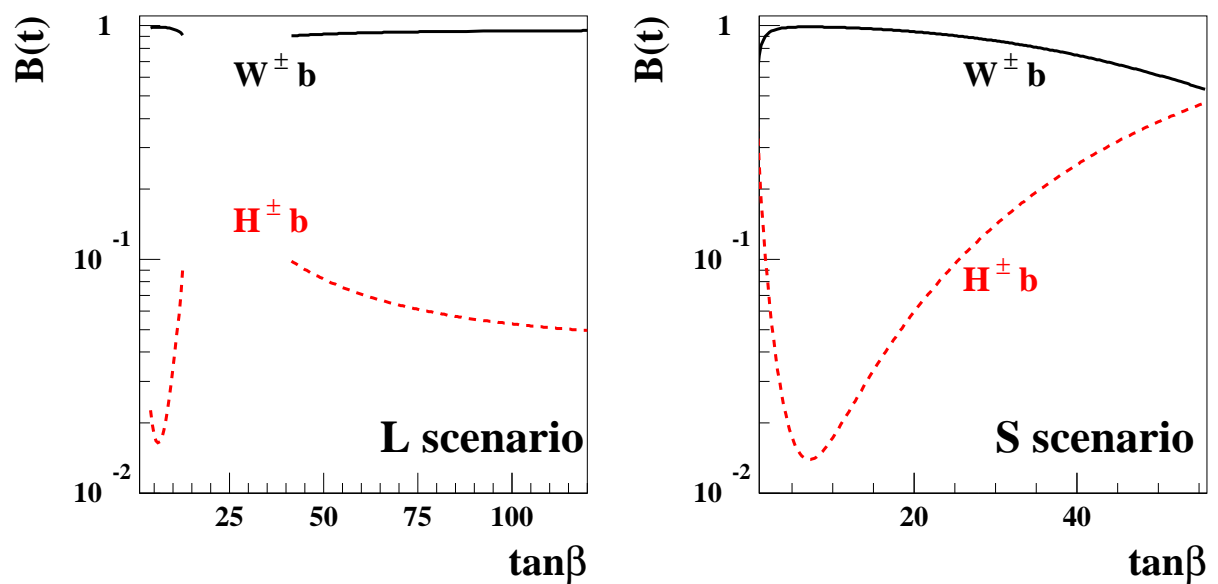

Figure 7: The top-quark branching ratios in the $\mathbf{L}$ (left) and $\mathbf{S}$ (right) scenarios, defined in (13) and 14), respectively. 